\title{
Review Article: Multicarrier Communication for Underwater Acoustic Channel
}

\author{
Hamada Esmaiel, Danchi Jiang \\ School of Engineering, University of Tasmania, Hobart, Australia \\ Email: hamada.esmaiel@utas.edu.au, Danchi.Jiang@utas.edu.au
}

Received June 24, 2013; revised July 22, 2013; accepted July 26, 2013

Copyright (C) 2013 Hamada Esmaiel, Danchi Jiang. This is an open access article distributed under the Creative Commons Attribution License, which permits unrestricted use, distribution, and reproduction in any medium, provided the original work is properly cited.

\begin{abstract}
In past decades, there has been a growing interest in the discussion and study of using underwater acoustic channel as the physical layer for communication systems, ranging from point-to-point communications to underwater multicarrier modulation networks. A series of review papers were already available to provide a history of the development of the field until the end of the last decade. In this paper, we attempt to provide an overview of the key developments, both theoretical and applied, in the particular topics regarding multicarrier communication for underwater acoustic communication such as the channel and Doppler shift estimation, video and image transmission throw multicarrier techniques, etc. This paper also includes acoustic propagation properties in seawater and underwater acoustic channel representation.
\end{abstract}

Keywords: Underwater Communication; Multicarrier Communication; Channel Coding; Orthogonal Frequency Division Multiplexing (OFDM); Filterbank Multicarrier (FBMC)

\section{Introduction}

Underwater acoustic channels are considered to be "quite possibly nature's most unforgiving wireless medium" [1]. The complexity of underwater acoustic channels is dominated by the ocean environment characteristics which include significant delay, Double-side-spreading, Dopplerspreads, frequency-selective fading, and limited bandwidth [2]. However, efficient underwater communications are critical to many types of scientific and civil missions in the ocean, such as ocean monitoring, ocean exploration, undersea rescue, and undersea disaster response. Human knowledge and understanding of the oceans, rests on our ability to collect information from remote undersea locations. Together with sensor technology and vehicular technology, wireless underwater communications are desirable to enable new applications ranging from environmental monitoring to gathering of oceanographic data, marine archaeology, and search and rescue missions.

New technologies of high speed communication for image and video transmission are also desirable to facilitate the next generation of efficient undersea expeditions. However, current acoustic communication technologies can only provide limited data rates due to the particular physical features of channel [3]. The corresponding wireless technology for undersea communications still needs significant further development. Research has been ac- tive for over a decade on designing the methods for wireless information transmission underwater. Due to electromagnetic waves in underwater channel propagate only over extremely short distances acoustic wave used. In contrast, acoustic waves can propagate over much longer distances. However, an underwater acoustic channel presents a communication system designer with many difficulties.

Figure 1 [4] illustrates the scenario of shallow water multipath propagation. In such situation, in addition to the direct path, the signal also propagates via reflections from the surface and bottom, resulting in a multipath effect with much larger time dispersion that of wireless propagation in air.

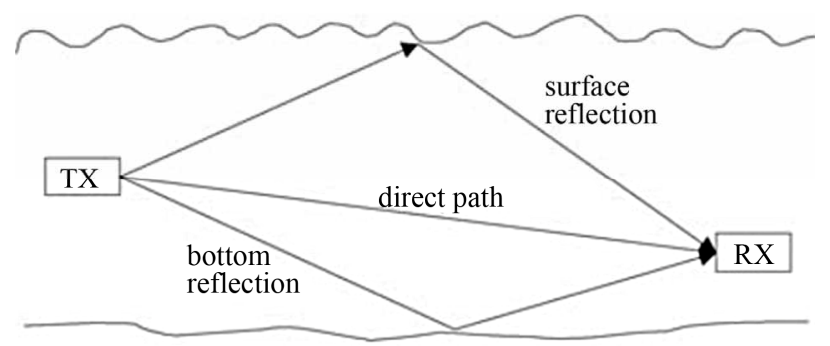

Figure 1. Shallow water multipath propagation: in addition to the direct path, the signal propagates via reflections from the surface and bottom. 
There are many issues that have to be carefully examined when designing an acoustic based transmission system for underwater channels. Some of these are [5]: 1) Attenuation due to the absorption of the acoustic waves in water, which limits the distance the sound, can travel; 2) Low propagation speed of the sound, roughly around $1500 \mathrm{~m} / \mathrm{s}$; 3) Multipath due to the reflection from the bottom and surface of sea, causing echoes and interference; 4) The transmitted signal suffers from the heterogeneous characteristics of the underwater channel as well as Doppler's effect caused by the movement of transmiter and receiver; 5) Noise in the ocean. The noise level can mask the portion of the signal and block the corresponding carried data.

The properties of the underwater medium are also extremely varied, and change in both space and time. Fluctuations due to environmental characteristics include seasonal changes, geographical variations both in temperature and salinity, seabed relief, currents, tides, internal waves, movement of the acoustic systems and their targets, etc. All this makes the underwater acoustic signal fluctuating randomly. As such, the selection of modulation and error correction techniques is very challenging. To mitigate the bandwidth limitation, multicarrier modulation is used in the underwater acoustic channel. It is an alternative to overcome the long-time delays in underwater acoustic channels. It increases the symbol interval and thereby decreases the inter-symbol interference (ISI) [6].

In this paper, we aim to provide a brief overview of the key developments, both theoretical and applied, in the field in the subsequent period. We also hope to provide an insight into some of the open problems and challenges facing researchers in this field in the near future. Rather than attempting to provide an exhaustive survey of all research in the field, we will concentrate on multicarrier modulation for underwater acoustic channel simulation, challenges, ideas and developments that are likely to be the keystone of future digital signal processing for underwater acoustic communication systems.

This paper summarizes several aspects of underwater acoustic communication. It is organized into the following sections. Section 2 briefly summarizes acoustic propagation properties in seawater. Section 3 analyses channel coding performance for multicarrier modulation in underwater acoustic communication systems. In Section 4, multicarrier modulations for underwater acoustic communications are presented. The underwater acoustic channel estimation for underwater communication is included in Section 5. In Section 6, Doppler shift estimation for underwater acoustic communications is studied. Image \& video transmission over the underwater acoustic channel (UWAC) with multicarrier modulation is discussed in Section 7. A summary of this paper is included in Section 8.

\section{Acoustic Propagation Properties in Seawater}

A good understanding and reasonably accurate modelling of the underwater acoustic channel is the required basis upon which all other works for underwater networks can be carried out. Several models are already available for calculating and predicting the attenuation [7,8], which can also help to model other aspects of the underwater acoustic channel. Furthermore, parameters of frequency, distance, depth, acidity to salinity, and temperature of the underwater environment can be used to characterize how the channel acts and how network can possibly perform. This section summarizes underwater channel models for that purpose.

\subsection{Propagation Loss}

The transmitted acoustic signal in underwater acoustic communication reduces strength with increasing distance due to many factors such as absorption caused by magnesium sulphate and boric acid, particle motion and geometrical spreading, etc. Propagation loss is composed mainly of three aspects, namely, geometrical spreading, attenuation and the anomaly of propagation. The latter is nearly impossible to model. However it is known that the signal attenuation, in $\mathrm{dB}$, that occurs over a transmission distance $l$ for a signal frequency $f$ can be approximated as [9]:

$$
10 \log A(1, f)=k \cdot 10 \log l+l \cdot 10 \log \alpha,
$$

where $\alpha$ is the absorption coefficient in $\mathrm{dB} / \mathrm{km}$, which can be obtained from the particular models characterizing it, and $k$ represents the geometrical spreading factor with its value between $1-2$.

\subsection{Absorption Coefficient}

Attenuation by absorption occurs due to the conversion of acoustic energy into heat in sea-water. This process is frequency dependent since at higher frequencies more energy is absorbed. The attenuation by absorption models considered for inclusion into the Thorp model [9]. Equation (2) provides the absorption coefficient in $\mathrm{dB} / \mathrm{km}$ as a function in carrier frequency $f_{c}$ :

$$
10 \log \alpha=\frac{0.1 f_{c}^{2}}{1+f_{c}^{2}}+\frac{40 f_{c}^{2}}{4100+f_{c}^{2}}+2.75 \times 10^{-4} f_{c}^{2}+0.003
$$

\subsection{Ambient Noise Model}

Ambient noise in the ocean can be described as Gaussian and having a continuous power spectral density (p.s.d.) [9]. The four most prominent sources for ambient noise are the turbulence, shipping, wind driven waves and thermal noise. Their p.s.d. in $\mathrm{dB}$ re $\mu \mathrm{Pa}$ per $\mathrm{Hz}$ are given 
by the formulae Equations (3)-(6) shown below, respectively [9]:

$$
\begin{gathered}
10 \log N_{t}(f)=17-30 \log f, \\
10 \log N_{s}(f)=40+20(s-0.5)+26 \log f \\
-60 \log (f+0.03)
\end{gathered}
$$

$10 \log N_{w}(f)=50+7.5 \sqrt{\omega}+20 \log f-40 \log (f+0.4)$,

$$
10 \log N_{t h}(f)=-15+20 \log f .
$$

The ambient noise in the ocean is affected by different factors in specific frequency ranges. In the noise models given in Equations (3) to (6), the effect of colored noise denoted by $N_{t}(f)$ represents the turbulence noise at frequency $f, N_{s}(f)$ the shipping noise (with $S$ as the shipping factor which lies between 0 and 1), $N_{w}(f)$ the wind driven wave noise (with $\omega$ as the wind speed in $\mathrm{m} / \mathrm{s}$ ), and $N_{t h}(f)$ the thermal noise. The composite noise p.s.d. can obtained in $\mu \mathrm{Pa}$ from [9]:

$$
N(f)=N_{t}(f)+N_{s}(f)+N_{w}+N_{t h}(f) .
$$

\subsection{Signal-to-Noise Ratio}

In UWAC, signal-to-noise ratio can be calculated [10] based on signal attenuation and the noise p.s.d., Specifically, the SNR observed at the receiver can be calculated in $\mu \mathrm{Pa}$ re $\mathrm{dB}$ per $\mathrm{Hz}$ using the following equation:

$$
\operatorname{SNR}(l, f)=\frac{P}{A(l, f) N(f) \Delta f},
$$

where $\operatorname{SNR}(l, f)$ is the SNR over a distance $l$ and a transmission center frequency $f, P$ is the signal transmission power and $\Delta f$ represents the receiver noise bandwidth. Equation (8) clearly shows that the underwater acoustic channel SNR is a function of transmission frequency. As such we can find the optimal frequency for UWAC to maximize SNR. The attenuation-noise (AN) factor, given by $A(l, f)$ can be used to reflect the frequency dependent part of the SNR. By close analysis of this relationship, it can also be used to determine the optimal frequency at which the maximal narrow-band SNR is achieved for each transmission distance $l$.

Since the SNR is inversely proportional to the attenuation-noise factor, the optimal frequency is that for which the value of $1 / \mathrm{AN}$ (represented in $\mathrm{dB}$ re $\mu \mathrm{Pa}$ per $\mathrm{Hz}$ ) is the highest over the combination of a certain distance, $f_{o}(l)$. In Figure 2, [10] frequency-dependent part of the narrowband SNR, $1 / A(1, f) N(f)$, is shown. In this plot the attenuation and noise parameters are selected as $k=1.5, S=0.5$, and $\omega=0$, to reflect the UWAC with moderate shipping activity and no wave noise.

It can be seen that there is a frequency for which the

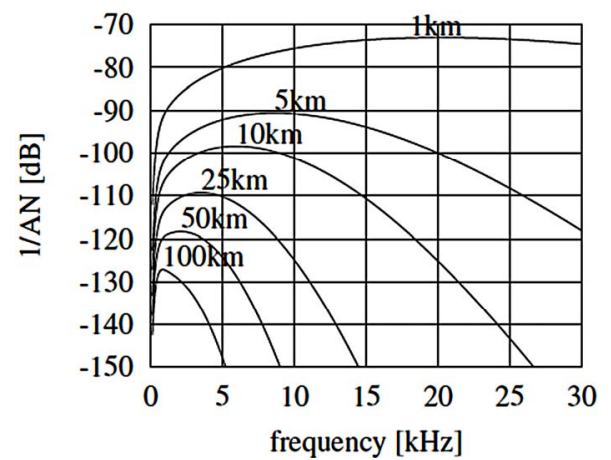

Figure 2. Narrowband SNR, $1 / A(1, f) N(f) ; k=1.5 ; s=0 ; \omega$ $=\mathbf{0}$.

narrowband SNR is maximized for a particular distance, for the given attenuation and noise constants. This optimal frequency, let it denoted $f_{o}(l)$, can be selected as the carrier frequency $f_{c}$ for that particular transmission distance [10].

\subsection{Channel Capacity}

As per the Shannon theorem the channel capacity i.e. the theoretical upper bound on data that can be communicated through a undistorted channel subject to additive white Gaussian noise is given by the following formula [9]:

$$
C=B \log _{2}\left(1+\frac{S}{N}\right),
$$

where $B$ is the channel bandwidth in $\mathrm{Hz}$ and $S / N$ represents the channel SNR. The basic Shannon relationship shown in Equation (9) can be extended to be applicable in cases where the noise is dependent on frequency to take the form of [9]:

$$
C=\int_{B} \log _{2}\left(1+\frac{S(f)}{N(f)}\right) \mathrm{d} f .
$$

For time-invariant channel, if a certain time interval with Gaussian noise, we can obtain the total capacity by dividing the total bandwidth into multiple narrow subbands and summing their individual capacities collectively. Then each sub-band has a small width $\Delta f$ which is centered on the transmission frequency, i.e. the bandwidth. In the case where the transmission bandwidth, $B(l)$, over a distance $l$ is known along with the transmission power $P(l)$, we can extend Equation (10) to obtain the channel capacity over a distance $l$ [9]:

$$
C=\int_{B} \log _{2}\left(\frac{P(l)}{A(l, f) N(f) B(l)}\right) \mathrm{d} f .
$$

The choice of the underlying absorption coefficient model imposes a dependence of the capacity on depth, temperature, salinity and acidity as well. 


\subsection{Doppler Shift and UWAC Multipath}

In underwater communication, the relative movement between the transmitter and the receiver due to the constant motion of nodes results in Doppler shifts, which significantly distort received signals. It is required to estimate the Doppler shift and compensate it for all UWAC applications. Different from the case of terrestrial communication where the Doppler effect is modeled by a frequency shift, due to the slow sound speed in water, the effect of transceiver motion on the duration of the symbol cannot be neglected [11]. Doppler phase $\varphi_{d}$, depending on the relative velocity $v$ and the ratio between the carrier frequency $f_{c}$ and the symbol rate $R=1 / T$ [12] caused in the received signal as:

$$
\varphi_{d}=-2 \pi f_{c} T \frac{\Delta}{1-\Delta}=-2 \pi \frac{f_{c}}{R} \frac{v}{c-v} .
$$

From $[13,14]$, UWAC multipath representation for multipath arrival $p$ is characterized by its mean magnitude gains $\alpha_{p}$ and delay $t_{p}$. These quantities are dependent on the path length $l_{p}$, which in turn is a function of the given range $R$.

The path magnitude gain is given by $\alpha_{p}=\Gamma_{p} / \sqrt{A\left(l_{p}, f_{c}\right)}$, where $\Gamma_{p}=(1 / \sqrt{2})^{r_{p}}$ is the amount of loss due to reflection at the bottom and surface, and $r_{p}$ is the number of reflections for path $p$. From Equation (1), the acoustic propagation loss, represented by $\left(l_{p}, f_{c}\right)$, resulting in the following equation:

$$
A\left(l_{p}, f_{c}\right)=l_{p}^{k}\left[\alpha\left(f_{c}\right)\right]^{l_{p}} .
$$

The delay for path $p$, given by $t_{p}=l_{p} / c \quad(c=1500$ $\mathrm{m} / \mathrm{s}$ is the speed of sound in water) and $l_{p}$ is the path length for path $p$ [14]. $r_{p}=0,1,3,5,7$ for each path respectively, the path lengths can be calculated using planar geometry.

\subsection{Underwater Acoustic Noise Model}

An empirical model for the noise of the acoustic underwater channel in shallow water from the analysis of field data measurements has been presented in [15]. In that paper, a probability density function for the noise amplitude distribution is proposed and the associated likelihood functions are derived. As a result, an expression to the probability of symbol error for binary signaling is presented for the channel. In Addition, the results of simulations conducted using the field collected noise samples are presented, in order to verify the noise effect on the performance of underwater acoustic communication binary signaling systems. The analysis of field data measurements has shown that the noise amplitude distribution presents good fitting with the Student's $t$ distribution. From [15], the symbol error probability of the bi- nary UWAN channel can be expressed as:

$$
P_{e}=k \sqrt{2 E_{b} / N_{o}} \int_{0}^{\infty}\left[\left(4 E_{b} / N_{o}\right)(x+1)^{2}+5\right]^{-2.75} \mathrm{~d} x .
$$

where $x$ is a vector of $M$ discrete amplitude levels of noise, integer $k=1,2, \cdots, M$, and $E_{b} / N_{o}$ is the energy per bit to noise power spectral density ratio.

\subsection{Underwater Acoustic Channel Simulator}

The signal transmission in underwater acoustic communications can be modeled as a time-varying channel. In particular, the noise-free signal at a receiver is described as a convolution [16]:

$$
y(t)=\int_{-\infty}^{\infty} h(t, \tau) s(t-\tau) \mathrm{d} \tau, t \in[0, T s],
$$

between the channel impulse response $h(t, \tau)$ and the source signal $s(t)$, where $T_{s}$ is the signal duration. This description of linear time-variant systems is quite generic. In underwater acoustic communications, other variants of the description may also be used. For example, for a channel with discrete multipath components, the model [17-19] used, and UWA channel represented as:

$$
c(\tau, t)=\sum_{p} A_{p}(t) \delta\left(\tau-\tau_{p}(t)\right) .
$$

Within a data block of interest, each path delay can be associated with one Doppler scale factor as:

$$
\tau_{p}(t)=\tau_{p}-a_{p} t,
$$

and the path amplitudes are assumed constant within one data block $A_{p}(t) \approx A_{p}$. Furthermore we assume that the UWA channel can be well approximated by $N_{p}$ dominant discrete paths. Hence, the channel model can be simplified to be:

$$
c(\tau, t)=\sum_{p=1}^{N_{p}} A_{p} \delta\left(\tau-\left[\tau_{p}-a_{p} t\right]\right) .
$$

\section{Channel Coding Performance for Multicarrier Modulation in Underwater Acoustic Communication}

It is required that digital communication systems, particularly for underwater use, to perform accurately and reliably in the presence of noise and interference. Among many possible ways to achieve this goal, forward error correction coding is the most effective and economical. The fast temporal variations, long multipath delay spreads, and severe frequency-dependent attenuations of underwater acoustic communication channels are extremely complex that impedes underwater acoustic data transmission. To alleviate this problem, channel coding is indispensable in UWA communication system to increase the 
reliability [20]. Also for underwater communication commonly used symbol demodulation schemes do not depend on the noise power, so bit error performance without error correcting coding will not be improved [21]. Here, Forward Error Correction (FEC) is a type of error correction which improves simple error detection schemes by enabling the receiver to correct errors once they are detected. This reduces the need for retransmissions and energy consumption [22].

The power of error correcting codes increases with the channel coding length constraint and approaching the Shannon limit with a large number of length constraints. But in return the complexity of the decoder also increases with length constraint [23]. For these reasons, it is desirable to construct long codes and minimize the complexity of the decoder. The concatenation of codes is a cheap solution for that [24] for multicarrier modulation in underwater acoustic communication. The major drawback of the concatenation code is that the decoder is unable to decode correctly in the presence of a burst of erroneous bits. Hence, an interleaver can be designed to introduce a dependency between the bits input. In order to minimize the error rate two evolved coding scheme [22], which are summarized in the following two subsections.

\subsection{Reed Solomon Coder}

Reed Solomon codes $(n, k, t)$ are cyclic codes, built from $n$ symbols with a maximum of $n=q-1$, where $q$ is the number of elements in the Galois Field $\left(G F^{q}\right)$ $\left(q=2^{n}\right)$ and $t$ is the power correcting code. So the number of control symbols is $2 t$.

It has been approached in [25] to develop a sufficiently robust acoustic link allowing the transmission of different information using Reed Solomon coder and conventional coding to protect data transmission over underwater acoustic channel. Where underwater acoustic link is designed to transmit different kinds of data as text, images and speech signal, blind spatial-temporal equalizer is used to reduce different underwater acoustic perturbations. To improve the underwater acoustic link performance and obtain a higher code rate, Reed Solomon Block Turbo Codes (RS BTC) has been introduced and tested in real conditions, with the aim to decrease the BER. A differential coding has been used to solve the phase ambiguities. Channels block coders algorithm applies to transmission of constant data. Where Block codes are FEC codes that enable a limited number of errors to be detected and corrected without retransmission. Block codes has been used to improve the performance of a communications system when other means of improvement (such increasing transmitter power or using a stronger modulator) are impractical [26].

\subsection{Low Density Parity Check Code (LDPC)}

LDPC codes are a special type of linear block coder. The parity-check matrix H of LDPC codes are very sparse, i.e. they can be specified by a matrix containing mostly 0 's [27]. It is used to reduce error codes and achieve credible transmit performance of underwater digital signal. Turbo code has also been recommended to apply to underwater digital speech communication system and simulation results in underwater digital speech system discussed in [27] have shown that LDPC has a better performance than the turbo coder.

Modification in the LDPC has been proposed to more matched multicarrier underwater communication systems [28], by focusing on the case of matching the coding symbols with the modulation symbols. Experimental results show, with real data that whenever the uncoded BER is below 0.1 , normally no decoding errors will occur for the rate $1 / 2$ of nonbinary LDPC codes used in [28] and it consistent with the simulation results. The unencoded BER can serve as a quick performance indicator to assess how likely the decoding will succeed. The researcher results show that LDPC code system has a better error correct performance and can achieve a better BER under the relative lower SNR [20].

Performances of LDPC codes with different parameters over different underwater acoustic communication channels are studied [20], by adjusting the encoding and decoding parameters according to different underwater acoustic channels.

When LDPC coder used as channel coder for zeropadding orthogonal frequency division multiplexing ( $\mathrm{ZP}$ OFDM) multicarrier modulation the spectral efficiency $\alpha$ and the data rate $R$ are [29,30]:

$$
\begin{gathered}
\alpha=\frac{T}{T+T_{g}} \cdot \frac{\left|S_{D}\right|}{K} \cdot r \cdot \log _{2} M \text { bits } / \mathrm{s} / \mathrm{Hz}, \\
R=\alpha B \mathrm{~kb} / \mathrm{s} .
\end{gathered}
$$

where $T$ is the ZP-OFDM symbol duration, $T_{g}$ is the guard interval, $K$ is number of all subcarrier, $\left|S_{D}\right|$ is data subcarrier in total, $r$ is the rate of nonbinary LDPC code [28], $M$ is the quadrature amplitude modulation symbol, and $B$ is the channel bandwidth. LDPC produces high block-error-rate (BLER) performance in $\mathrm{ZP}$ OFDM multi-carrier system.

\section{Multi-Carrier Modulation for Underwater Acoustic Communications}

Multi-carrier modulation Systems are well known to be attractive for communications through multi-path communications channels. The traditional approach expects the symbol duration of the transmitted signal to be larger than channel delay spread. This results in a low rate 
"sampling" of the channel impulse response (once per symbol in each sub-channel) and a sub-channel bandwidth that is less than the coherence bandwidth of the physical propagation channel [31]. Multi-Carrier Modulation (MCM) have become popular in UWA channel for two reasons. First, a signal can be processed in a receiver without the increase of noise or interference caused by linear equalization of a single carrier signal; second, the long symbol period used in MCM ensures greater immunity to impulse noise and fast fades [32].

\subsection{Orthogonal Frequency Division Multiplexing}

Multicarrier modulation in the form of orthogonal frequency division multiplexing (OFDM) has been studied and implemented for broadband wired and wireless communications for the past two decades. OFDM is widely adopted because of a number of its advantages as [33-35]: 1) Orthogonality of subcarrier signals that allows: a) Easy generation of transmit signal through an inverse fast Fourier transform (IFFT) block; b) Easy separation of the transmitted data symbols at the receiver through a fast block; c) Easy equalization through a scalar gain per subcarrier; d) Easy adoption to multiple-input multipleoutput (MIMO) channels; 2) Closely spaced orthogonal subcarriers partition the available bandwidth into a maximum collection of narrow sub-bands; 3) Adaptive modulation schemes can be applied to subcarrier bands to maximize bandwidth efficiency/transmission rate; 4) The very special structure of OFDM symbols simplifies the tasks of carrier and symbol synchronizations.

OFDM as an MCM is particularly efficient when noise is spread over a large portion of the available bandwidth. It transmits signals over multiple orthogonal sub-carriers simultaneously and performs robustly in severe multipath environments achieving high spectral efficiency. OFDM used in underwater communications as a superior alternative to single carrier broadband modulation to achieve high data rate transmission $[4,17,36,37]$. It has been proved to be an effective technique for combating the multipath delay spread without the need for complex time-domain equalizers, due to its robustness against frequency selective fading and narrowband interference. In fact, if the number of subcarriers is large enough, each subcarrier only deals with flat fading rather than with frequency selective fading as a wideband carrier does. The narrowband interference will affect only one or two subcarriers of the whole bunch of subcarriers. The major issue in applying OFDM to underwater channels is the motion induced Doppler distortion which creates nonuniform frequency offset in a wideband acoustic signal $[4,17,36,37]$.

\subsubsection{ZP-OFDM for Underwater Acoustic Communication}

Zero-padded (ZP) $[29,30,38]$ orthogonal frequency division multiplexing (OFDM) has been extensively investigated for high data rate underwater acoustic communication. Frequency-domain oversampling method [30] is used to avoid information loss incurred by the overlap-add operation. A larger FFT size used to improve system performance over underwater acoustic channels with significant Doppler spread. The system is validated using real data collected from field experiments. Zero padding is used instead of the conventional cyclic prefix in order to save transmission power during the (long) guard interval. ZP-OFDM transmitted and received signals in the time domain during underwater channel paths signals after implemented to make zero-pad guard interval accept channel path delay shown in Figure 3 [30].

For ZP-OFDM With symbol duration $T$, the subcarrier spacing is $\Delta f=1 / T$, and the subcarriers are located at frequencies

$$
f_{k}=f_{c}+\frac{k}{T}, \quad k=\frac{-K}{2}, \cdots, \frac{K}{2-1},
$$

where $f_{c}$ is the center frequency, and $K$ is the total number of subcarriers, leading to the bandwidth $B=K / T$. $s[k]$ denote the information symbol on the $k^{\text {th }}$ subcarrier. The transmitted passband signal is [30]:

$$
\tilde{x}(t)=2 \operatorname{Re}\left(\sum_{k \in S_{A}} s[k] \mathrm{e}^{j 2 \pi f_{k} t} g(t)\right), \quad t \in\left[0, T^{\prime}\right],
$$

where $T^{\prime}$ is the ZP-OFDM block duration as shown in Figure 3.

The baseband signal $z(t)$ is obtained with the passband to baseband downshifting and the lowpass filtering, leading to [30]:

$$
\begin{aligned}
z(t)= & \sum_{p=1}^{N_{p}} A_{p} \mathrm{e}^{j 2 \pi f_{c}\left(b_{p} t-\tau_{p}\right)} \\
& \cdot \sum_{k \in S_{A}} s[k] \mathrm{e}^{j 2 \pi m / k\left(\left(1+b_{p}\right) t-\tau_{p}\right)} g\left(\left(1+b_{p}\right) t-\tau_{p}\right),
\end{aligned}
$$
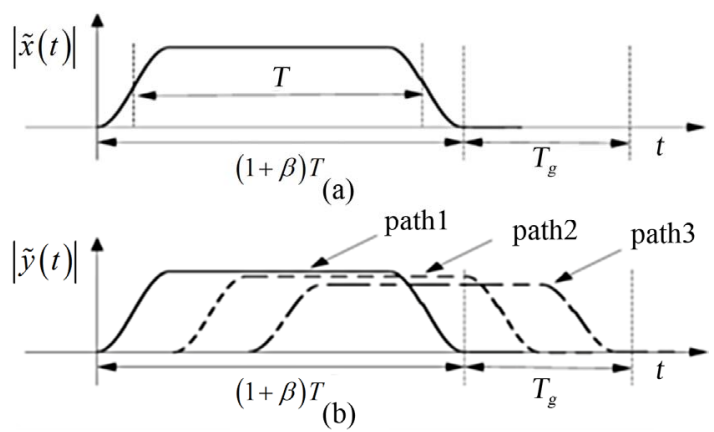

Figure 3. Illustration of the transmitted and received signals in the time domain. (a) One transmitted ZP-OFDM block; (b) One received ZP-OFDM block. 
where $b_{p}$ represents the residual Doppler rate satisfying:

$$
1+b_{p}=\frac{1+a_{p}}{1+\hat{a}} .
$$

ZP-OFDM saves Power transmitted and increases channel capacity. In [39] authors derived bounds to the channel capacity of OFDM systems over the underwater (UW) acoustic fading channel as a function of the distance between the transmitter and the receiver.

\subsubsection{Pilot Signal Design for OFDM Multicarrier Transmission}

The challenges in pilot design for multicarrier transmission over underwater acoustic time-varying channels are two-fold [40,41]: 1) Sets of adjacent observations are needed to estimate the inter-carrier interference (ICI) coefficients; 2) Keeping pilot and data symbols orthogonal at the receiver is challenging due to the ICI. Generally there are three types of pilot insertion methods for OFDM system, comb-type; block-type; hexagonal gridtype, etc. [42]. Random selection of pilot subcarriers is motivated by the compressive sensing [43] with the systematic use of pilot blocks in regular intervals as seen in non-sparse channel estimation of time-varying channels [29].

In [40], authors are interested in how to address the ICI between data and pilot subcarriers without guard zeros. As it decreases spectral efficiency, focus has been put on whether data symbol carrying subcarriers should be used as observations in channel estimation, which also contain ICI originating from the pilot symbols. The performance under varying amounts of pilot overhead has also been studied. Specifically, authors are looking for an optimum tradeoff between using more pilots or a more robust modulation scheme, to achieve the highest spectral efficiency.

\subsubsection{OFDM Based on Discrete Cosine Transform for Underwater Acoustic Communication}

The orthogonal feature of conventional OFDM can also be achieved by inversing DCT (IDCT)-DCT structure for underwater communication, which reduces implementation area and increases computational speed, as only real calculations, is required. This system provides higher peakto-average power ratio (PAPR) reduction and achieves better noise immunity and hence a better bit error rate (BER) performance than standard OFDM, while maintaining a low implementation cost $[32,44]$.

DCT based OFDM is a better technology for underwater acoustic communication, because the bandwidth required for DCT is half of that required for DFT when both systems have same number of subcarriers which will be matched with underwater channel limited band- width. It has also been shown that the speed of calcultion of orthogonal components is increased three folds while the implementation size reduces to half as compared to fast FFT based design [44]. Furthermore, it is known that the DCT basis have excellent spectral compaction and energy concentration properties which in turn lead to improved performance with suitable channel estimation [44]. As DCT is widely adopted in image/ video coding standards, by using it for modulation/demodulation on frequency selective channels it will result in a better integrated system design and a reduced overall implementation cost [44].

\subsubsection{Orthogonal Signal-Division Multiplexing for Underwater Acoustic Communication}

Orthogonal signal-division multiplexing (OSDM) is proposed as a UWA communication system scheme that measures the multipath profile without an adaptation or interpolation process, to achieve stable communication in doubly spread channels [45]. The performance compareson of the OSDM scheme and existing schemes in doubly spread channels has been done. The ill-conditioned problem exists for conventional OSDM, which employs a single transducer in the receiver. The introduction of a multichannel receiver has been found to be effective against the ill-conditioned problem. Evaluation of OSDM communication done by comparing it to existing schemes with single-carrier Recursive Least Square Differential Feedback Equalizer (RLS-DFE) [46] and OFDM. OSDM with a multichannel receiver is attractive in terms of communication quality. It achieves much better BER performance comparing to the other schemes in both static and dynamic channels, although its complexity is less than that of RLS-DFE. OSDM can become a viable alternative offering a highly reliable communication environment for UWA communication with multipath and Doppler spread (tested only for shallow water) with practical complexity [45] and it can be a very important of point for future research.

\subsubsection{Time Domain Synchronous Orthogonal Frequency Division Multiplexing}

A time domain synchronous orthogonal frequency division multiplexing (TDS-OFDM) scheme with dual Pseudonoise (PN) sequence [47] is proposed in [48] for UWA communication. TDS-OFDM system is used for underwater acoustic channel shown in Figure 4 [48].

Instead of using cyclic prefix (CP) or zero padding (ZP) as the guard interval, the proposed TDS-OFDM scheme uses two identical PN sequences as guard interval, and utilizes them for frame synchronization and channel estimation. TDS-OFDM increases the spectrum efficiency over the conventional CP or ZP OFDM systems where additional pilots have to be inserted for channel estima- 


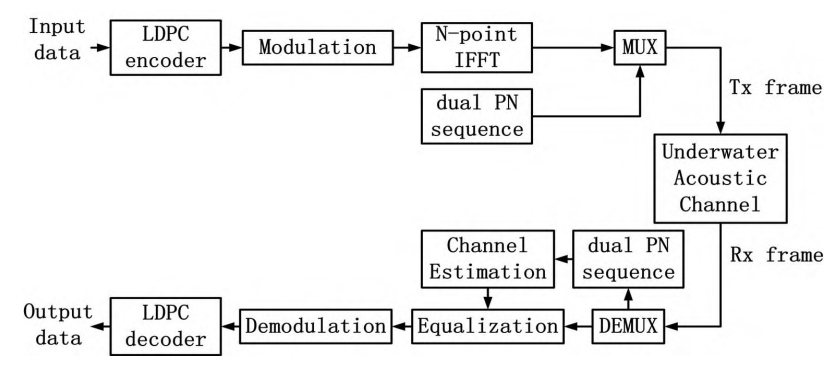

Figure 4. TDS-OFDM system for underwater acoustic channel.

tion. With the dual PN at each frame header, compressed sensing channel estimation is adopted and a rather simple equalization design to reduce the receiver complexity.

Conventional performance of ZP-OFDM receiver is severely limited by the ICI due to the fast channel variations within each OFDM symbol. Furthermore, the UWA channel is wideband in nature due to the small ratio of the carrier frequency to the signal bandwidth [30,49]. In $[50,51]$ cyclic shift keying spread spectrum OFDM method propose as a UWA communication system.

In [50,51] cyclic shift keying spread spectrum OFDM method is proposed to use in a UWA communication system. The aim is to solving the problem of low data rate of direct sequence spread spectrum underwater acoustic communication [52] and that of the complexity of the receivers of M-ary spread spectrum. The paper also aimed to improve bandwidth efficiency and bit rates. This method has high data rate comparing to conventional direct sequence spread spectrum for the underwater acoustic communication and low bit error rate.

\subsubsection{Power and Bit Loading for Underwater Acoustic OFDM System}

Adaptive bit and power loading is a constraint optimization problem with generally two cases of practical interest, where the objectives are the achievable data rate maximization (RM) and system margin maximization (MM) [53].

In $[41,54,55]$ researchers propose a different optimization model for underwater acoustic (UWA) channels, which is achieved by two algorithms: one is the bandwidth-efficient bit loading algorithm; the other is the Lloyd algorithm based limited feedback procedure. It aims at minimizing the power consumption under constraints of the constant symbol data rate and desired biterror-rate (BER). Algorithms are employed to quantize the CSI at the receiver and construct the codebook, which is adopted to achieve the limited feedback process. After selecting an initial bit loading vector upon the current CSI, the receiver will broadcast its index to the transmitter, then the transmitter will compute the bandwidthefficient bit loading algorithm and allocate the corresponding power and bits to each OFDM subcarrier. Also algorithms are used for UWA cooperative communication system, which involves the Decode-and-Forward (DF) transmission protocol $[41,56]$.

The ambient noise power $\omega\left(f_{i}\right)$ for each OFDM sub-carrier can be proposed as $[41,56]$ :

$$
\omega\left(f_{i}\right)=\int_{f_{i L}}^{f_{i H}} N\left(f_{i}\right) d\left(f_{i}\right)=\int_{f_{i L}}^{f_{i H}} 10^{\frac{N_{0}}{10}} \cdot f_{i}^{-\frac{9}{5}} d\left(f_{i}\right),
$$

where $f_{i L}$ and $f_{i H}$ are the bound of ith sub-carrier frequency. The maximum capacity of a UWA SISOOFDM system with total system transmits power constraint describe as $[41,56]$ :

$$
\begin{aligned}
& \max _{\varepsilon_{i}} C=\sum_{i=1}^{N} \Delta f \cdot \log _{2}\left(1+\frac{\varepsilon_{i} g_{i}}{\Gamma}\right) \\
& \text { s.t. } \quad \sum_{i=1}^{N} \varepsilon_{i} \leq \varepsilon_{s}, \quad \varepsilon_{i} \geq 0, i=1, \cdots, N
\end{aligned},
$$

$N$ is the number of sub-channels, $\Delta f$ is the subchannel transmitted bandwidth, $\varepsilon_{i}$ is the transmitted power in the ith sub-channel, $\varepsilon_{s}$ the total transmitted power, $g_{i}$ is the sub-channel power gain and the power loading will be based on this parameter, and $g_{i}=\left|H_{i}\right|^{2} / \sigma_{i}^{2}$, where $\left|H_{i}\right|$ is the fading amplitude of the ith sub-channel, $\left|H_{i}\right|^{2}$ is the sub-channel power, $\sigma_{i}^{2}$ is the ambient noise power and it's a constant of ith sub-channel, and $\varepsilon_{i} g_{i}$ is the $S N R$ or carrier-to-noise ratio $(C N R)$ per sub-channel. $\Gamma$ is the "SNR gap" for characterizing the difference between the $S N R$ needed to achieve a certain data rate for a practical system and the theoretical limit.

\subsubsection{OFDM Receiver Designs for Underwater Acoustic Communication}

OFDM receiver designs for underwater acoustic channels with user and path specific Doppler scaling distortions were proposed in [57]. The method was motivated by the cooperative communications framework [58], where distributed transmitter/receiver pairs may experience significantly different Doppler distortions, as well as by the single user scenarios, where distinct Doppler scaling factors may exist among different propagation paths. The conventional approach of front end resampling that corrects common Doppler scaling may not be appropriate in such scenarios, rendering a post FFT signal that is contaminated by user and path-specific inter-carrier interference. To counteract this problem, authors propose a family of front end receiver structures that utilize multiple resampling (MR) [59] branches, with each matched to the Doppler scaling factor of a particular user and path. Following resampling, FFT modules transform the Doppler shift compensated signals into the frequency domain for further processing through linear or nonlinear detection schemes. As part of the overall receiver structure, a 
gradient descent approach is also proposed to refine the channel estimates obtained by standard sparse channel estimators.

\subsection{Filterbank Multicarrier for Underwater Communications}

In order to combat time dispersion of UWA channels, it has been proposed to deploy OFDM technique with a sufficiently long CP. Moreover, to keep the bandwidth efficiency of the transmission high, long OFDM symbols that are at least four times of the length of the CP should be used. Due to small sideband power leakage, filter bank multicarrier techniques are considered as interesting alternatives to traditional OFDMs for spectrum pooling Cognitive Radio [60]. This leads to an OFDM system in which channel variation over each OFDM symbol may be unacceptably large, thus, results in a significant level of ICI [61]. For that filter bank multicarrier (FBMC) systems can be optimized for robust performance in the doubly dispersive UWA channels [61]. OFDM multicarrier losses bandwidth efficiency of the transmission due to the allocation of $20 \%$ of each OFDM symbol to its CP. This is equivalent of saying the CP length is one quarter of the length of each fast FFT block in the OFDM system. Moreover, since the length of CP should be at least equal to the duration of the channel impulse response, and the latter is usually very long in UWA channels, very long symbols is used in the OFDM systems for UWA communications [61]. On the other hand, to avoid ISI, the prototype filter $p(t)$ designed as Nyquist filter [62,63]. The design method proposed in [64] constructs an isotropic filter according to the equation.

$$
p(t)=\sum_{k=0}^{L} a_{k} h_{4 k}(t)
$$

where $h_{k}(t)$ are the set of Hermite functions defined as [61]:

$$
h_{n}(t)=\frac{1}{(2 \pi)^{n / 2}} \mathrm{e}^{\pi t^{2}} \frac{\mathrm{d}^{n}}{\mathrm{~d} t^{n}} \mathrm{e}^{-2 \pi t^{2}}
$$

In [61], it is noted that the presence of channel will result in a disturbed ambiguity function, $A_{p}^{d}(\tau, v)$, in which the null points of $A_{p}(\tau, v)$ are smeared out. Thus, it is argued that to design a robust prototype filter, the constraints on the nulls of the ambiguity function $A_{p}(\tau, v)$ may be relaxed. Each null point is replaced by a region in the $(\tau, v)$-plane, wich is termed a null region. It is thus proposed to design $p(t)$, one should choose to minimize the cost function:

$$
\zeta=\gamma_{0} \int_{A_{0}}\left|A_{p}(\tau, v)-1\right|^{2} \mathrm{~d} \tau \mathrm{d} v+\sum_{k=1}^{N} \gamma_{k} \int_{A_{k}}\left|A_{p}(\tau, v)\right|^{2} \mathrm{~d} \tau \mathrm{d} v \text {. }
$$

where $\gamma_{k}$ are sets of positive weighting factors, $A_{0}$ is the region around $(\tau, v)=(0,0)$ over which the peak of $A_{p}(\tau, v)$ remains approximately equal to one, and $A_{k}$ for $k=1,2, \cdots, N$, are sets of null regions.

UWA communication method using a class of FBMC systems was proposed [61]. This class of FBMC systems was designed to be robust against dispersions in time and frequency domain. When the Filterbank multicarrier technique is compared with OFDM, it clearly shows that there is a wide gap between the performance of FBMC and OFDM for underwater communication in saving bandwidth [61]. For the Single-user communications case OFDM offers a lower complexity. FBMC offers higher bandwidth efficiency. For Multi-input multi-output (MIMO) communications case, OFDM provides full flexibility. FBMC can be used in certain MIMO setups. Only FMT can offer the same flexibility as OFDM. But, FMT suffers from the same bandwidth loss as OFDM [65]. FBMC for underwater communication still have high poor research paper and need more research interest.

\subsection{MIMO for Underwater Acoustic Channel}

Multi-input multi-output (MIMO) techniques have been extensively discussed in underwater acoustic communications to overcome the bandwidth limitation of undersea channel [66]. Combined with OFDM modulation, MIMO techniques provide substantial spectral efficiency and reasonable robustness against frequency fading while keeping simple equalizer structure [67-70]. Long acoustic multipath, however, limits the applicability of MIMO channel estimation methods that require inversion of a matrix whose size is proportional to both the number of transmit elements and the multipath spread. To overcome this problem, an adaptive algorithm is used [71] that does not require matrix inversion and operates in a decision-directed manner, thus reducing both the computational complexity and the overhead. Reduction in complexity has been sought through selection of significant impulse response coefficients which results in a reduced-size matrix inversion [72-74]. MIMO-OFDM design consists of the following key components: 1) Null subcarriers are inserted at the transmitter to facilitate the compensation of Doppler shifts at the receiver; 2) Pilot tones are used for MIMO channel estimation; and 3) An iterative receiver structure is adopted that couples MIMO detection with channel decoding [66].

OFDM has a number of desirable features, including low complexity of implementation and mature technologies that keep it as the dominant technology for single-user (point-to-point) underwater communications. Moreover, while OFDM can be easily adopted for MIMO channels, development of MIMO-FBMC systems/networks is still nontrivial and may be very limited. Only FMT, the less bandwidth-efficient member of the class of FBMC systems, can offer a similar level of flexibility as 
OFDM in MIMO channels. Therefore, the poor frequency spectra of subcarrier signals in OFDM are the main issue that limits the applicability of OFDM in some present and future development of broadband underwater communication systems. FBMC [75], on the other hand, is an elegant method scope with that by taking a filtering approach to underwater multicarrier communications.

\section{Underwater Acoustic Channel Estimation}

To reduce computation complexity of signal processing and improve the accuracy of symbol detection, receiver structures that are matched to the physical-feedback equalizer is designed first in [76], which rely on an adaptive channel estimator for its parameters computation. The channel estimation complexity is reduced in size by selecting only the significant components, whose delay span is often much shorter than the multipath spread of the channel. This estimation is used to cancel the postcursor ISI prior to the linear equalizer involved. Optimal coefficient selection is performed by truncation in magnitude. The advantages of this approach are the number reduction in receiver parameters, optimal implementation of sparse feedback, and efficient parallel implementation of adaptive algorithms for the multichannel pre-combiner, fractionally spaced channel estimators and the short feed forward equalizer filters [77].

Coherent modulation schemes such as phase shift keying (PSK) along with adaptive decision feedback equalizers (DFE) for spatial diversity used as an effective way of communication in such channels [78,79]. However, the long delay spread and rapid time variation of the channel often makes this approach computationally too complex for real-time implementations [80]. Although the underwater channel has a long impulse response, the multipath arrivals are often separated. This introduces the possibility of using a sparse equalizer with tap placement based on the actual channel response. This can potentially dramatically reduce the number of required taps and hence leads to a lower complexity, faster channel tracking and an enhanced performance [81].

In [81], the authors have proposed an algorithm to track the channel explicitly and determine the tap placement for the DFE based on this channel estimate. The equalizer and the channel estimator are updated individually throughout the packet. The channel estimator can also update either the whole estimation or a set of selected channel coefficients at one time in batch, depending on computational and channel considerations [82].

The channel estimation algorithms can cope with spatial diversity by multi-channel combining before equalization. Adaptive estimation is performed using minimum mean square error as the overall optimization criterion. The receiver is implemented in a multichannel configu- ration, which provides the array processing gain necessary for many of the underwater acoustic channels. The complexity of the detection algorithm is linear in the number of receive elements and in dependent on the modulation level of the transmitted signals [83]. DFE structures may suffer from error propagation due to the feedback of possible erroneous decisions in the loop.

Hence powerful forward error correction (FEC) codes are needed to ensure low bit error rate (BER) communication. Turbo codes are a class of powerful codes that utilize iterative information exchange between two decoders to correct errors. Inspired by this idea, researchers have developed turbo equalization techniques where iterative interactions between the equalizer and a decoder result in joint estimation, equalization and decoding [84].

A new channel estimation [85] equalization and phase correction scheme has been developed. The new scheme estimates the acoustic fading channel without separating the phase drift and phase rotation for each symbol and then the SIMO receive signals are equalized and combined. Finally the phase drift/rotation of symbols is corrected per group of symbols using estimated average phase drift/rotation.

Authors in [29] considered sparse channel estimation using subspace methods and compressed sensing on channels subject to moderate Doppler effects, and extended the compressed sensing receivers to handle channels with different Doppler scales on different paths. Channel estimation and efficient symbol detection studied in [86] where the design of state-of-the-art training sequences and sparse learning via iterative minimization (SLIM) algorithm was proposed to achieve sparse channel estimation. The authors have developed a conjugate gradient (CG) based detector, which exploits the diagonalization properties of the circulant channel matrix to significantly improve the performance of multi-input multi-output (MIMO) underwater acoustic communications (UAC).

The UWA communication system under consideration employs orthogonal frequency division multiplexing (OFDM) and receiver preprocessing to compensate for the Doppler effects before channel estimation. First extend the original homotopic algorithm from real-valued signals to the complex valued ones. Then propose two enhancements to the sparse recovery-based UWA channel estimator by exploiting the UWA channel temporal correlations, including the use of a first-order GaussMarkov model and the recursive least-squares algorithm for channel tracking. Moreover the authors propose a scheme to optimize the pilot placement over the OFDM subcarriers based on the discrete stochastic approximation.

Sparse channel estimation for multicarrier underwater acoustic communication were proposed in [29]. Based on the path-based channel model, subspace methods are 
well-known techniques from the array-processing literature for the channel estimation problem. Also recent compressed sensing technique is employed to develop some new methods, namely Orthogonal Matching Pursuit (OMP) and Basis Pursuit (BP) [87,88]. Based on the continuous time characterization of the path delays, finer delay has been suggested to be used of resolution over complete dictionaries. Also the compressed sensing receivers can be extended to handle channels with different Doppler scales on different paths, supplying ICI pattern estimates that can be used to equalize the ICI [29].

Using extensive numerical simulation and experimental results, researchers find that, in comparison to the LS receiver, the subspace methods show significant performance improvements on channels that are sparse, but perform worse if most received energy comes from diffuse multipath. To improve LS estimation, simple windowing and dewindowing technique has been used to improve the accuracy of an existing basis expansion model (BEM) and develop a windowed least-squares (WLS) estimator for doubly-selective fading channels [89]. The compressed sensing algorithms do not suffer this drawback, and benefit significantly from the increased time resolution using sophisticated dictionaries. When accounting for different Doppler scales on different paths, BP can effectively handle channels with very large Doppler spread.

\section{Doppler Shift Estimation for Underwater Acoustic Communications}

In underwater acoustic transmissions, Doppler effects can be caused by propagation medium movements or by the relative velocity between the transmitter and the receiver [12]. It often causes additional difficulties for the processing of received signals. For instance, underwater acoustic communications with submarines navigating at speeds up to $25 \mathrm{knots}(12.8 \mathrm{~m} / \mathrm{s})$ is challenging, due to large Doppler range that the receiver must be able to cope with. Doppler effects result in a compression/expansion of the transmitted signal [90]. At the receiver side, it is desirable to remove the effect of these phenomena before any further processing, such as timing and carrier recovery. A receiver which performs optimal phase synchronization and channel equalization jointly has been suggested for underwater communication system [91].

Based on cross-correlation among training sequences located at the beginning and at the end of transmitted data frame, an algorithm is developed for Doppler shift estimation [12]. From these training sequences two phase estimates are calculated: a coarse one and a precise one. The precise estimation has much smaller ambiguity range than the coarse one, but it yields higher precision for the estimated Doppler frequency. Authors have shown that a convenient combination of the coarse and precise phases leads to a good Doppler shift estimation within the speed range under consideration in the particular application discussed. Frequency-dependent Doppler shifts caused by the low carrier frequency of the underwater acoustic channel communication.

Depending on the null subcarrier of the ZP-OFDM, multi-carrier facilitates Doppler compensation, and the pilot subcarriers used in channel estimation [17] where an application of OFDM is investigated in wideband UWA channels with nonuniform Doppler shifts. To compensate for the nonuniform Doppler distortion, a twostep approach was used by resampling followed by highresolution uniform compensation of the residual Doppler. Also based on that, a block-by-block receiver is suitable for fast-varying channels. Based on the availability of pilot tones in the OFDM transmission, a method developed to estimate the offset parameters, and a hybrid channel estimator was then proposed to combine the offset compensated [92].

Compressed Sensing (CS) can be developed as a method to solve the channel estimation problems for an UWA system. Based on the existence of a sparse representation of the treated signal and an over-complete dictionary with a set of non-orthogonal bases, in [93] a new type of channel estimator using the compressed sensing theory is proposed, leading to a sparse channel estimation from the highly dependence on Doppler compensation. Instead of using various compensation approaches, the proposed index is designed by modeling Doppler shifts as the atoms shifts in over-complete dictionary. From the results, this method improves the mean square error (MSE) performance with lower complexity and hardware cost. In addition, the method has the additional benefit being less sensitive to Doppler rate variety.

\section{Multicarrier Communication for Image \& Video Transmission over Underwater Acoustic Channel}

Recently, there has been a growing interest in development and deployment of image and video transmission techniques for underwater communication networks for scientific, environmental, commercial, and military purposes $[94,95]$. High speed underwater image transmission capabilities can enable the next generation of undersea expeditions. Efficient image transmission over the band limited underwater channels relies on two aspects [96]: 1) Efficient data compression, and 2) Bandwidth efficient modulation.

The compressed sensing technique [97] generates minimum amount of information necessary for transmission which makes it useful in underwater communication. The 
combination of compressed sensing and nonlinear analog processing can also been employed as joint source and channel coding [3]. Underwater multimedia sensor networks (UMSNs) [98] have been proposed and drawn the immediate attention in the research community. However, the practical implementation of these currently designed and envisioned applications directly depends on reliability and quality-aware communication capabilities of the deployed UMSNs. Comprehensive performance evaluation of error concealment and error correction algorithms for quality-aware image transmission over UMSNs is reported in [99].

For high-speed image transmission using Multicarrier modulation $[100,101]$ MMSE based equalization with the placement of a pilot symbol for very of three sub-carrier payload resulted in good performance close to the ideal performance of the equalization. Set Partitioning in Hierarchical Trees (SPIHT) is an efficient wavelet-based progressive image-compression technique, designed to minimize the mean-squared error (MSE) between the original and decoded imagery used for highly compression technique [102-105]. Since underwater acoustic channel suffers from significant bit error rates, some mechanism to protect the encoded image is required.

16-HQAM used as mapper for ZP-OFDM, for unequal error protection using HQAM modulation technique it produce highly capability of highly speed image transmission over UWAC. Multicarrier communication system can transmit high speed image without equalizer and also with small overhead forward error correction bits. System depend on the guard interval, large zero-pad guard interval of ZP-OFDM system used to avoid information loss incurred by the overlap-add operation to improve system performance over underwater acoustic channels with large Doppler spread [106,107].

The real-time wireless video transmission from an underwater vehicle to a surface platform represents one of the last milestones in the development of autonomous systems for ocean exploration and monitoring. The goal of combining video transmission over UWAC approaches is to mitigate the gap between the bit rate needed for video transmission and that supported by the acoustic channel. Konstantinos Pelekanakis [108] developed a video transmission technique over underwater acoustic channel using coherent single carrier modulation technique for phase detection. For that a high rate acoustic link for underwater video transmission was implemented, where image encoding was accomplished using the JPEG DCT, scalar quantization and run-length Huffman encoding. Transmitter processing includes signal constellations.

A system employing variable rate M-QAM techniques was designed and applied to the experimental data transmitted over a short vertical channel. Excellent results were obtained at bit rates up to $150 \mathrm{kbps}$, using modula- tion methods with bandwidth efficiency as high as 6 $\mathrm{bits} / \mathrm{sec} / \mathrm{Hz}$. Such rate is sufficient to support real-time transmission of compressed video. The feasibility of realtime video transmission over short horizontal acoustic links was addressed in [109] where the standard MPEG4 video compression technique and a wavelet-based method were combined with acoustic transmission based on coded OFDM modulation to study the feasibility of video transmission using an acoustic system for deep-sea oilfield supervisory control and inspection. The waveletbased encoder algorithm includes techniques that deal with spatial and temporal redundancies in video sequences. In contrast to MPEG-4 compression, spatial redundancies are exploited by applying the Discrete Wavelet Transform (DWT) to each of the frames composing the video sequence. A motion compensation algorithm [110] to reduce temporal redundancies has been incorporated, which is a core functionality of the MPEG-4 encoder. The codec is based on the popular Differential Pulse Code Modulation (DPCM) model, which is widely used in video compression standards. Its main components are the DWT multilevel decomposition of the input frame using the bi-orthogonal wavelet, quantization of the DWT coefficients using Vector Quantization (VQ). The images are smoother due to the high quantization ratio for the high subbands of the wavelet decomposition and, as expected, no blocking effect is noticed at all [109].

\section{Summary \& Conclusion}

In this paper we surveyed multicarrier communication techniques used for data transmission over the underwater acoustic channel up to as the major aspects can be summarized as: 1) Underwater channel model and its challenging in multicarrier communication modulation like OFDM and FBMC summarized and detail reviewed; 2) The advances of underwater acoustics technology applications and areas emerged in; 3) Channel characteristics and channel models in reducing noise and channel noise estimates; 4) Multicarrier modulation and multiinput multi-output techniques detailed description for underwater communication; 5) Doppler estimation and underwater channel estimation challenging discuses and techniques used evaluated and summarized; 6) Performance evaluation for different channel coder used for underwater acoustic multicarrier modulation; 7) Changing and used techniques for high speed image and video transmission over UWAC also summarized and discussed.

\section{REFERENCES}

[1] D. Brady and J. C. Preisig, "Underwater Acoustic Communications," In: H. V. Poor and G. W. Wornell, Eds., Wireless Communications: Signal Processing Perspectives, Vol. 8, Prentice-Hall, Inc., Upper Saddle River, 1998, pp. 330-379. 
[2] A. C. Singer, J. K. Nelson and S. S. Kozat, "Signal Processing for Underwater Acoustic Communications," IEEE Communications Magazine, Vol. 47, No. 1, 2009, pp. 9096. doi:10.1109/MCOM.2009.4752683

[3] I. Iglesias, A. Song, J. Garcia-Frias, M. Badiey and G. R. Arce, "Image Transmission over the Underwater Acoustic Channel via Compressive Sensing," 45th Annual Conference on Information Sciences and Systems, Baltimore, 23-25 March 2011, pp. 1-6.

[4] M. Stojanovic, "Underwater Wireless Communications: Current Achievements and Research Challenges," IEEE Oceanic Engineering Society Newsletter, Vol. 41, No. 1, 2006, pp. 10-13.

[5] Y. Labrador, M. Karimi, D. Pan and J. Miller, "Modulation and Error Correction in the Underwater Acoustic Communication Channel," International Journal of Computer Science and Network Security, Vol. 9, No. 7, 2009, pp. 123-130.

[6] S.-J. Hwang and P. Schniter, "Efficient Communication over Highly Spread Underwater Acoustic Channels," International Conference on Mobile Computing and Networking, Montreal, 9-14 September 2007, pp. 11-18.

[7] F. De Rango, F. Veltri and P. Fazio, “A Multipath Fading Channel Model for Underwater Shallow Acoustic Communications," IEEE International Conference on Communications, Ottawa, 10-15 June 2012, pp. 3811-3815.

[8] L. M. Wolff, E. Szczepanski and S. Badri-Hoeher, "Acoustic Underwater Channel and Network Simulator," $O C$ EANS, Yeosu, 21-24 May 2012, pp. 1-6.

[9] A. Sehgal, I. Tumar and J. Schönwälder, "AquaTools: An Underwater Acoustic Networking Simulation Toolkit," OCEANS, Sydney, 24-27 May 2010, pp. 1-10.

[10] M. Stojanovic, "On the Relationship between Capacity and Distance in an Underwater Acoustic Communication Channel," ACM SIGMOBILE Mobile Computing and Communications Review, Vol. 11, No. 4, 2007, pp. 34-43. doi: $10.1145 / 1347364.1347373$

[11] A. Fish, S. Gurevich, R. Hadani, A. Sayeed and O. Schwartz, "Delay-Doppler Channel Estimation with Almost Linear Complexity," 2012, in press.

[12] J. Trubuil and T. Chonavel, "Accurate Doppler Estimation for Underwater Acoustic Communications," OCEANS, Yeosu, 21-24 May 2012, pp. 1-5.

[13] M. Vajapeyam, S. Vedantam, U. Mitra, J. C. Preisig and M. Stojanovic, "Time Cooperative Schemes for Underwater Acoustic Communications," IEEE Journal of Oceanic Engineering, Vol. 33, No. 4, 2008, pp. 489-501. doi:10.1109/JOE.2008.2005338

[14] Y. Chen, X. Xu, L. Zhang and Z. Zou, "Design and Application of Dynamic Coding in Shallow Water Acoustic Communications," OCEANS, Yeosu, 21-24 May 2012, pp. 1-6.

[15] J. S. Panaro, F. R. Lopes, L. M. Barreira and F. E. Souza, "Underwater Acoustic Noise Model for Shallow Water Communications," Presented at the Brazilian Telecommunication Symposium, 2012.

[16] L. Chunshan, Y. V. Zakharov and C. Teyan, "Doubly Selective Underwater Acoustic Channel Model for a Mov- ing Transmitter/Receiver," IEEE Transactions on Vehicular Technology, Vol. 61, No. 3, 2012, pp. 938-950. doi:10.1109/TVT.2012.2187226

[17] B. Li, S. Zhou, M. Stojanovic, L. Freitag and P. Willett, "Multicarrier Communication over Underwater Acoustic Channels with Nonuniform Doppler Shifts," IEEE Journal of Oceanic Engineering, Vol. 33, No. 2, 2008, pp. 198-209. doi:10.1109/JOE.2008.920471

[18] S. Yerramalli and U. Mitra, "On Optimal Resampling for OFDM Signaling in Doubly-Selective Underwater Acoustic Channels," OCEANS, Quebec City, 11-15 September 2008, pp. 1-6.

[19] G. L. Stüber, "Principles of Mobile Communication," Springer, New York, 2011.

[20] X. Xiaomei, C. Yougan, Z. Lan and F. Wei, "Comparison of the Performance of LDPC Codes over Different Underwater Acoustic Channels," 12th IEEE International Conference on Communication Technology, Nanjing, 1114 November 2010, pp. 155-158.

[21] C. R. Berger, W. Chen, S. Zhou and J. Huang, "A Simple and Effective Noise Whitening Method for Underwater Acoustic Orthogonal Frequency Division Multiplexing," The Journal of the Acoustical Society of America, Vol. 127, No. 4, 2010, pp. 2358-2367. doi:10.1121/1.3309452

[22] N. Nasri, L. Andrieux, A. Kachouri and M. Samet, "Efficient Encoding and Decoding Schemes for Wireless Underwater Communication Systems," 7th International Multi-Conference on Systems Signals and Devices, Amman, 27-30 June 2010, pp. 1-6.

[23] M. D. Haque, S. E. Ullah and M. R. Ahmed, "Performance Evaluation of a Wireless Orthogonal Frequency Division Multiplexing System under Various Concatenated FEC Channel-Coding Schemes," 11th International Conference on Computer and Information Technology, Khulna, 24-27 December 2008, pp. 94-97.

[24] M. Chitre, S. Shahabudeen, L. Freitag and M. Stojanovic, "Recent Advances in Underwater Acoustic Communications \& Networking," OCEANS, Quebec City, 11-15 September 2008, pp. 1-10.

[25] J. Trubuil, A. Goalic and N. Beuzelin, "Synchronization and Channel Coding in Shallow Water Acoustic Communication," OCEANS, Quebec City, 11-15 September 2008, pp. 1-5.

[26] J. Trubuil, A. Goalic and N. Beuzelin, "An Overview of channel Coding for Underwater Acoustic Communications," Military Communications Conference, Orlando, 29 October-1 November 2012, pp. 1-7.

[27] W. Han, J. Huang and M. Jiang, "Performance Analysis of Underwater Digital Speech Communication System Based on LDPC Codes," 4th IEEE Conference on Industrial Electronics and Applications, Xi'an, 25-27 May 2009, pp. 567-570.

[28] J. Huang, S. Zhou and P. Willett, "Nonbinary LDPC Coding for Multicarrier Underwater Acoustic Communication," IEEE Journal on Selected Areas in Communications, Vol. 26, No. 9, 2008, pp. 1684-1696. doi:10.1109/JSAC.2008.081208

[29] C. R. Berger, S. Zhou, J. C. Preisig and P. Willett, "Sparse Channel Estimation for Multicarrier Underwater 
Acoustic Communication: From Subspace Methods to Compressed Sensing," IEEE Transactions on Signal Processing, Vol. 58, No. 3, 2010, pp. 1708-1721. doi:10.1109/TSP.2009.2038424

[30] Z. Wang, S. Zhou, G. B. Giannakis, C. R. Berger and J. Huang, "Frequency-Domain Oversampling for Zero-Padded OFDM in Underwater Acoustic Communications," IEEE Journal of Oceanic Engineering, Vol. 37, No. 1, 2012, pp. 14-24. doi:10.1109/JOE.2011.2174070

[31] A. K. Morozov and J. C. Preisig, "Underwater Acoustic Communications with Multi-Carrier Modulation," OCEANS, Boston, 18-21 September 2006, pp. 1-6.

[32] P. Kumar and P. Kumar, "Performance Evaluation of DFT-Spread OFDM and DCT-Spread OFDM for Underwater Acoustic Communication," IEEE Vehicular Technology Conference, Quebec City, 3-6 September 2012, pp. 1-5.

[33] B. Farhang-Boroujeny, "OFDM Versus Filter Bank Multicarrier," IEEE Signal Processing Magazine, Vol. 28, No. 3, 2011, pp. 92-112. doi:10.1109/MSP.2011.940267

[34] R. van Nee and R. Prasad, "OFDM for Wireless Multimedia Communications," Artech House, Inc., Norwood, 2000.

[35] Y. G. Li, "Orthogonal Frequency Division Multiplexing for Wireless Communications," Springer-Verlag, New York, 2009.

[36] B. Li, S. Zhou, M. Stojanovic, L. Freitag, J. Huang and P. Willett, "MIMO-OFDM over an Underwater Acoustic Channel," OCEANS, Vancouver, 29 September-4 October 2007, pp. 1-6.

[37] A. Thottappilly, "OFDM for Underwater Acoustic Communication," Master of Science, Virginia Polytechnic Institute and State University, Blacksburg, 2011.

[38] B. Li, S. Zhou, M. Stojanovic, L. Freitag and P. Willet, "Multicarrier Underwater Acoustic Communications over Fast-Varying Channels," IEEE Journal of Oceanic Engineering, Vol. 33, No. 2, 2008, pp. 198-209. doi:10.1109/JOE.2008.920471

[39] C. Polprasert, J. A. Ritcey and M. Stojanovic, "Capacity of OFDM Systems over Fading Underwater Acoustic Channels," IEEE Journal of Oceanic Engineering, Vol. 36, No. 4, 2011, pp. 514-524. doi:10.1109/JOE.2011.2167071

[40] C. R. Berger, J. Gomes and J. M. Moura, "Study of Pilot Designs for Cyclic-Prefix OFDM on Time-Varying and Sparse Underwater Acoustic Channels," OCEANS, Santander, 6-9 June 2011, pp. 1-8.

[41] X. Huang, "Capacity Criterion-Based Power Loading for Underwater Acoustic OFDM System with Limited Feedback," IEEE International Conference on Wireless Communications, Networking and Information Security, Beijing, 25-27 June 2010, pp. 54-58.

[42] T. Pedersen and A. Bloomberg, "OFDM Pilot-Aided Underwater Acoustic Channel Estimation," International Journal of Electrical, Electronics and Computer Systems, Vol. 9, No. 1, 2012, pp. 1-3.

[43] R. G. Baraniuk, "Compressive Sensing," IEEE Signal Processing Magazine, Vol. 24, No. 4, 2007, pp. 118-121. doi:10.1109/MSP.2007.4286571
[44] P. Kumar, "DCT Based OFDM for Underwater Acoustic Communication," 1st International Conference on Recent Advances in Information Technology, Dhanbad, 15-17 March 2012, pp. 170-176.

[45] T. Ebihara and K. Mizutani, "Underwater Acoustic Communication With an Orthogonal Signal Division Multiplexing Scheme in Doubly Spread Channels," IEEE Journal of Oceanic Engineering, Vol. PP, No. 99, 2013, pp. 1-12. doi:10.1109/JOE.2013.2245273

[46] M. Stojanovic, "Recent Advances in High-Speed Underwater Acoustic Communications," IEEE Journal of Oceanic Engineering, Vol. 21, No. 2, 1996, pp. 125-136. doi:10.1109/48.486787

[47] R. Mutagi, "Pseudo Noise Sequences for Engineers," Electronics \& Communication Engineering Journal, Vol. 8, No. 2, 1996, pp. 79-87.

[48] H. Jinxing, Y. R. Zheng, W. Jintao and S. Jian, "Dual PN Padding TDS-OFDM for Underwater Acoustic Communication," Oceans, Hampton Road, 14-19 October 2012, pp. 1-4.

[49] Z. Wang, S. Zhou, J. Catipovic and P. Willett, "Asynchronous Multiuser Reception for OFDM in Underwater Acoustic Communications," IEEE Transactions on Wireless Communications, Vol. 12, No. 3, 2013, pp. 1-12.

[50] L. Y. Jing and J. G. Huang, "Cyclic Shift Keying Spread Spectrum OFDM Method over Underwater Acoustic Channel," IEEE International Conference on Signal Processing, Communication and Computing, Hong Kong, 12-15 August 2012, pp. 798-801.

[51] J.-G. Huang, C.-B. He, J. Han and Q.-F. Zhang, "Cyclic Shift Keying Spread Spectrum Underwater Acoustic Communication," Acta Physica Sinica, Vol. 58, No. 12, 2009, pp. 8379-8385.

[52] E. Sozer, J. Proakis, R. Stojanovic, J. Rice, A. Benson and M. Hatch, "Direct Sequence Spread Spectrum Based Modem for Under Water Acoustic Communication and Channel Measurements," OCEANS, Seattle, 13-16 September 1999, pp. 228-233.

[53] N. Papandreou and T. Antonakopoulos, "Bit and Power Allocation in Constrained Multicarrier Systems: The Single-User Case," EURASIP Journal on Advances in Signal Processing, Vol. 2008, No. 1, 2008, p. 11.

[54] X. Huang and V. B. Lawrence, "Bandwidth-Efficient Bit and Power Loading for Underwater Acoustic OFDM Communication System with Limited Feedback," IEEE 73rd Vehicular Technology Conference, Yokohama, 1518 May 2011, pp. 1-5.

[55] L. Yang, L. Zhou and M. Yu, "Adaptive Bit Loading Algorithm for OFDM Underwater Acoustic Communication System," International Conference on Electronics and Optoelectronics, Dalian, 29-31 July 2011, pp. 350- 352.

[56] H. Xiaopeng and L. Yong, "Capacity Criterion-Based Power Allocation for OFDM Cooperative Underwater Acoustic Communications with Limited Feedback," OCEANS, Hampton Road, 14-19 October 2012, pp. 1-4.

[57] K. Tu, T. M. Duman, M. Stojanovic and J. G. Proakis, "Multiple-Resampling Receiver Design for OFDM over Doppler-Distorted Underwater Acoustic Channels," IEEE Journal of Oceanic Engineering, Vol. 38, No. 2, 2013, pp. 
333-346. doi:10.1109/JOE.2012.2221812

[58] M. Uysal, "Cooperative Communications for Improved Wireless Network Transmission: Framework for Virtual Antenna Array Applications," Information Science Reference, University of Waterloo, Waterloo, 2010.

[59] K. Tu, "Multi-Carrier Communications over Underwater Acoustic Channels," Doctor of Philosophy, Arizona State University, Phoenix, 2011.

[60] Q. Zhang, A. B. Kokkeler and G. J. Smit, "An Oversampled Filter Bank Multicarrier System for Cognitive Radio," 19th International Symposium on Personal, Indoor and Mobile Radio Communications, Cannes, 15-18 September 2008, pp. 1-5. doi:10.1109/PIMRC.2008.4699865

[61] P. Amini, C. Rong-Rong and B. Farhang-Boroujeny, "Filterbank Multicarrier for Underwater Communications," 49th Annual Allerton Conference on Communication, Control, and Computing, Monticello, 28-30 September 2011, pp. 639-646. doi:10.1109/Allerton.2011.6120228

[62] B. Le Floch, M. Alard and C. Berrou, "Coded Orthogonal Frequency Division Multiplex," Proceedings of the IEEE, Vol. 83, No. 6, 1995, pp. 982-996. doi:10.1109/5.387096

[63] M. Alard, "Construction of a Multicarrier Signal," CA Patent CA 2,220,107, 1996.

[64] P. Amini, C. H. Yuen, R.-R. Chen and B. FarhangBoroujeny, "Isotropic Filter Design for MIMO Filter Bank Multicarrier Communications," IEEE Sensor Array and Multichannel Signal Processing Workshop, Jerusalem, 4-7 October 2010, pp. 89-92. doi:10.1109/SAM.2010.5606775

[65] L. Toni, L. Rossi, N. Agoulmine and J.-G. Fontaine, "Virtual UEP for Progressive Image Transmission in Underwater Communication," 2009, in press.

[66] L. Baosheng, H. Jie, Z. Shengli, K. Ball, M. Stojanovic, L. Freitag and P. Willett, "MIMO-OFDM for High-Rate Underwater Acoustic Communications," IEEE Journal of Oceanic Engineering, Vol. 34, No. 4, 2009, pp. 634-644. doi:10.1109/JOE.2009.2032005

[67] D. B. Kilfoyle, J. C. Preisig and A. B. Baggeroer, "Spatial Modulation Experiments in the Underwater Acoustic Channel," IEEE Journal of Oceanic Engineering, Vol. 30, No. 2, 2005, pp. 406-415. doi:10.1109/JOE.2004.834168

[68] S. Roy, T. M. Duman, V. McDonald and J. G. Proakis, "High-Rate Communication for Underwater Acoustic Channels Using Multiple Transmitters and Space-Time Coding: Receiver Structures and Experimental Results," IEEE Journal of Oceanic Engineering, Vol. 32, No. 3, 2007, pp. 663-688. doi:10.1109/JOE.2007.899275

[69] R. F. Ormondroyd, "A Robust Underwater Acoustic Communication System Using OFDM-MIMO," OCEANS, Aberdeen, 18-21 June 2007, pp. 1-6.

[70] P. Bouvet and A. Loussert, "An Analysis of MIMOOFDM for Shallow Water Acoustic Communications," OCEANS, Waikoloa, 19-22 September 2011, pp. 1-5.

[71] M. Stojanovic, "MIMO OFDM over Underwater Acoustic Channels," IEEE 43rd Asilomar Conference on Signals, Systems and Computers, Piscataway, 1-4 November 2009, pp. 605-609.

[72] Y. Li, N. Seshadri and S. Ariyavisitakul, "Channel Esti- mation for OFDM Systems with Transmitter Diversity in Mobile Wireless Channels," IEEE Journal on Selected Areas in Communications, Vol. 17, No. 3, 1999, pp. 461471. doi:10.1109/49.753731

[73] H. Minn and V. K. Bhargava, "An Investigation into Time-Domain Approach for OFDM Channel Estimation," IEEE Transactions on Broadcasting, Vol. 46, No. 4, 2000, pp. 240-248. doi:10.1109/11.898744

[74] M. Stojanovic, "OFDM for Underwater Acoustic Communications: Adaptive Synchronization and Sparse Channel Estimation," IEEE International Conference on Acoustics, Speech and Signal Processing, Las Vegas, 31 March4 April 2008, pp. 5288-5291.

[75] B. Saltzberg, "Performance of an Efficient Parallel Data Transmission System," IEEE Transactions on Communication Technology, Vol. 15, No. 6, 1967, pp. 805-811. doi:10.1109/TCOM.1967.1089674

[76] C. A. Belfiore and J. H. Park Jr., "Decision Feedback Equalization," Proceedings of the IEEE, Vol. 67, No. 8, 1979, pp. 1143-1156. doi:10.1109/PROC.1979.11409

[77] M. Stojanovic, L. Freitag and M. Johnson, "Channel-Estimation-Based Adaptive Equalization of Underwater Acoustic Signals," OCEANS, Seattle, 13-16 September 1999, pp. 590-595.

[78] R. Coates, "Underwater Acoustic Communications," $O C$ EANS, Vol. 3, 1993, pp. 420-425.

[79] M. Stojanovic, "Underwater Acoustic Communication," Wiley Encyclopedia of Electrical and Electronics Engineering, 1999.

[80] M. Chitre, S. Shahabudeen and M. Stojanovic, "Underwater Acoustic Communications and Networking: Recent Advances and Future Challenges," Marine Technology Society Journal, Vol. 42, No. 1, 2008, pp. 103-116. doi: $10.4031 / 002533208786861263$

[81] P. Shukla and L. Turner, "Channel-Estimation-Based Adaptive DFE for Fading Multipath Radio Channels," IEE Proceedings of Communications, Speech and Vision, Vol. 138, No. 6, 1991, pp. 525-543.

[82] P. Zarrinkhat, M. Ardakani and R. Yazdani, "Channel Estimation Considerations for Iterative Decoding in Wireless Communications," IEEE International Conference on Communications, Glasgow, 24-28 June 2007, pp. 1006-1011.

[83] E. Calvo and M. Stojanovic, "Efficient Channel-Estimation-Based Multiuser Detection for Underwater CDMA Systems," IEEE Journal of Oceanic Engineering, Vol. 33, No. 4, 2008, pp. 502-512.doi:10.1109/JOE.2008.2005355

[84] J. G. Proakis, E. M. Sozer, J. A. Rice and M. Stojanovic, "Shallow Water Acoustic Networks," IEEE Communications Magazine, Vol. 39, No. 11, 2001, pp. 114-119. doi: $10.1109 / 35.965368$

[85] Y. R. Zheng, "Channel Estimation and Phase-Correction for Robust Underwater Acoustic Communications," IEEE Military Communications Conference, Orlando, 29-31 October 2007, pp. 1-6.

[86] J. Ling, X. Tan, T. Yardibi, J. Li, H. He and M. L. Nordenvaad, "Enhanced Channel Estimation and Efficient Symbol Detection in MIMO Underwater Acoustic Com- 
munications," 43rd Asilomar Conference on Signals, Systems and Computers, Pacific Grove, 1-4 November 2009, pp. 600-604.

[87] K. Taehyuk and R. A. Iltis, "Matching Pursuits Channel Estimation for an Underwater Acoustic OFDM Modem," IEEE International Conference on Acoustics, Speech and Signal Processing, Las Vegas, 31 March-4 April 2008, pp. 5296-5299.

[88] J. Huang, C. R. Berger, S. Zhou and J. Huang, "Comparison of Basis Pursuit Algorithms for Sparse Channel Estimation in Underwater Acoustic OFDM," OCEANS, Sydney, 24-27 May 2010, pp. 1-6.

[89] Q. Fengzhong and Y. Liuqing, "On the Estimation of Doubly-Selective Fading Channels," IEEE Transactions on Wireless Communications, Vol. 9, No. 4, 2010, pp. 1261-1265. doi:10.1109/TWC.2010.04.080631

[90] B. S. Sharif, J. Neasham, O. R. Hinton and A. E. Adams, "A Computationally Efficient Doppler Compensation System for Underwater Acoustic Communications," IEEE Journal of Oceanic Engineering, Vol. 25, No. 1, 2000, pp. 52-61. doi:10.1109/48.820736

[91] M. Stojanovic, J. A. Catipovic and J. G. Proakis, "PhaseCoherent Digital Communications for Underwater Acoustic Channels," IEEE Journal of Oceanic Engineering, Vol. 19, No. 1, 1994, pp. 100-111. doi: $10.1109 / 48.289455$

[92] W. Zhaohui, Z. Shengli, J. C. Preisig, K. R. Pattipati and P. Willett, "Clustered Adaptation for Estimation of TimeVarying Underwater Acoustic Channels," IEEE Transaction on Signal Processing, Vol. 60, No. 6, 2012, pp. 3079-3091. doi:10.1109/TSP.2012.2189769

[93] H. Yu and J. Guo, "Compressed Sensing: Underwater Acoustic Channel Estimation with Doppler Shifts," 8th International Conference on Wireless Communications, Networking and Mobile Computing, Shanghai, 21-23 September 2012, pp. 1-4.

[94] I. F. Akyildiz, D. Pompili and T. Melodia, "Challenges for Efficient Communication in Underwater Acoustic Sensor Networks," ACM Sigbed Review, Vol. 1, No. 2, 2004, pp. 3-8. doi:10.1145/1121776.1121779

[95] E. M. Sozer, M. Stojanovic and J. G. Proakis, "Underwater Acoustic Networks," IEEE Journal of Oceanic Engineering, Vol. 25, No. 1, 2000, pp. 72-83. doi:10.1109/48.820738

[96] C. Pelekanakis, M. Stojanovic and L. Freitag, "High Rate Acoustic Link for Underwater Video Transmission," $O C$ EANS, Vol. 2, 2003, pp. 1091-1097.

[97] I. Iglesias, B. Lu, J. Garcia-Frias and G. Arce, "NonLinear Mappings for Transmission of Compressed Sensing Images," 48th Annual Allerton Conference on Communication, Control, and Computing, Allerton, 29 September-1 October 2010, pp. 726-732.

[98] S. A. Alkharabsheh and M. I. Younis, "Dynamics of MEMS Arches of Flexible Supports," Journal of Microelectromechanical Systems, Vol. 22, No. 1, 2013, pp. 216-224.

[99] P. Sarisaray-Boluk, V. Gungor, S. Baydere and A. Harmanci, "Quality Aware Image Transmission over Under- water Multimedia Sensor Networks," Ad Hoc Networks, Vol. 9, No. 7, 2011, pp. 1287-1301.

doi:10.1016/j.adhoc.2011.02.007

[100] J.-W. Han, S.-Y. Kim, K.-M. Kim, S.-Y. Chun and K. Son, "Design of OFDM System for High Speed Underwater Communication," International Conference on Computational Science and Engineering, Vancouver, 29-31 August 2009, pp. 988-992.

[101] T. B. Santoso and G. Hendrantoro, "Image Transmission with OFDM Technique in Underwater Acoustic Environment," 7th International Conference on Telecommunication Systems, Services, and Applications, Bali, 30-31 October 2012, pp. 37-41

[102] A. Said and W. A. Pearlman, "A New, Fast and Efficient Image Codec Based on Set Partitioning in Hierarchical Trees," IEEE Transactions on Circuits and Systems for Video Technology, Vol. 6, No. 3, 1996, pp. 243-250. doi:10.1109/76.499834

[103] C. Murphy and H. Singh, "Wavelet Compression with Set Partitioning for Low Bandwidth Telemetry from AUVs," Proceedings of the 5th ACM International Workshop on UnderWater Networks, Woods Hole, 30 September-1 October 2010, p. 1. doi:10.1145/1868812.1868813

[104] M. A. Kader, F. Ghani and R. B. Ahmed, "Unequal Error Protection for SPIHT Coded Image Transmission over Erroneous Wireless Channels," Asian Transactions on Fundamentals of Electronics, Communication \& Multimedia, Vol. 1, No. 5, 2011, pp. 1-6.

[105] B. Tomasi, L. Toni, P. Casari, J. Preisig and M. Zorzi, “A Study on the SPIHT Image Coding Technique for Underwater Acoustic Communications," Proceedings of the 6th ACM International Workshop on Underwater Networks, Seattle, 1-2 December 2011, pp. 1-9. doi: $10.1145 / 2076569.2076578$

[106] H. Esmaiel and D. Jiang, "Image Transmission over Underwater Acoustic Environment Using OFDM Technique with HQAM Mapper," 3rd IEEE International Conference on Information Science and Technology, Yangzhou, 23-25 March 2013, pp. 1596-1601.

[107] H. Esmaiel and D. Jiang, "SPIHT Coded Image Transmission over Underwater Acoustic Channel with Unequal Error Protection Using HQAM," 3rd IEEE International Conference on Information Science and Technology, Yangzhou, 23-25 March 2013, pp. 1365-1371.

[108] K. Pelekanakis, "Design and Analysis of a High-Rate Acoustic Link for Underwater Video Transmission," Master of Science, Massachusetts Institute of Technology, Cambridge, 2004

[109] J. Ribas, D. Sura and M. Stojanovic, "Underwater Wireless Video Transmission for Supervisory Control and Inspection Using Acoustic OFDM," OCEANS, Seattle, 2023 September 2010, pp. 1-9.

[110] D. F. Hoag, V. K. Ingle and R. J. Gaudette, "LowBit-Rate Coding of Underwater Video Using WaveletBased Compression Algorithms," IEEE Journal of Oceanic Engineering, Vol. 22, No. 2, 1997, pp. 393-400. doi: $10.1109 / 48.585958$ 